\title{
STUDY ON STRUCTURE AND ANTI-UV PROPERTIES OF SERICIN COCOONS
}

\author{
Bin Zhou ${ }^{1,2,3 \dagger}$, Huiling Wang ${ }^{1,2,3+*}$, Jumei Zhao ${ }^{1,3}$, Jiali Chen ${ }^{1,3}$, Hongtao Zhou ${ }^{1,3}$ \\ 1 School of Textiles and Clothing, Yancheng Polytechnic College, Yancheng 224005, China; sanlin2007@126.com \\ 2 College of Textile Science and Engineering (International institute of silk), Zhejiang Sci-Tech University, Hangzhou 310000, Chna;15949149207@126.com \\ 3 Jiangsu Province Engineering Research Center of Biomass Functional Textile Fiber Development and Application, Yancheng 224005, China \\ Corresponding Author. E-mail: 2007160107@yctei.edu.cn, sanlin2007@126.com; Tel.: +86-15949149207 \\ †These authors contributed equally to this work
}

\begin{abstract}
:
Through biogenetic engineering, the posterior silk glands of Bombyx mori larvae are degenerated, and only the middle silk glands are retained to secrete sericin to produce sericin fibers, so as to obtain sericin cocoons with high sericin content (>98.5\%). For this paper, sericin cocoons were studied, and the morphological structure characteristics and properties of sericin cocoons and common Bombyx mori cocoons were compared and analyzed through such modern test techniques as SEM, FTIR, and XRD. Results show that sericin cocoons have the basic morphological structure of cocoons, but because of the brittleness and hardness of sericin, the ssericin cocoons have poor integrity with a thermal decomposition rate that is higher than that of cocoons; the two cocoons are of a similar protein structure, with both $\alpha$-helix and $\beta$-sheet structures, and dominantly the $\beta$-sheet structure; and the crystallinity of sericin cocoons (10.48\%) is lower than that of the common Bombyx mori cocoons (27.10\%). Sericin was extracted from both cocoons to obtain a mixed sericin solution respectively, which were coated on base fabrics of polyester taffeta (210T) for coating finish and subjected to qualitative research for their anti-UV properties. The base fabrics coated with mixed sericin solutions of sericin cocoons and Bombyx mori cocoons have an anti-UV performance index (UPF) of $23.9 \%$ and $22.5 \%$ respectively, which are higher than that of the base fabric (8.9\%)..
\end{abstract}

\section{Keywords:}

sericin cocoon; sericin fiber; morphological structure; thermal property; anti-UV

\section{Introduction}

Silk is generally composed of fibroin and sericin. The sericin wraps the fibroin completely or partially and binds various layers of silk into a natural nonwoven compound material to assist silkworms in constructing the space for the growth and development of pupae, i.e., cocoons [1-7]. Studies have shown that the sericin outside the fibroin contains a mixture of pigment, grease, waxiness, and inorganic salts and plays a good role in protecting the fibroin to a certain extent [8, 9]. The cocoon can be divided into the cocoon coat, cocoon layer, and cocoon lining from outside to inside, and there is a significant difference in the content of sericin [10], so that the content of sericin in cocoon coat is close to 1.5 and 2 times that in the cocoon layer and cocoon lining respectively $[8,9$, 11-13]. Wild cocoons live outdoors, and their sericin content is higher [14-17]. The sericin content is as high as $15 \%$ to $25 \%$ in the cocoon coat, which is also associated with their better protection against UV rays to ensure the normal development of silkworm larvae $[18,19]$.

A silk reeling process is usually required before the production of silk products using cocoons, and one of the by-products obtained from silk reeling wastewater is sericin. The efficient extraction of sericin from silk reeling wastewater is a key step in the utilization of sericin. At present, the commonly used extraction methods, such as chemical coagulation, organic solvent, and acidification, have the disadvantages of long process flow and certain environmental pollution, and the centrifugation method and ultrafiltration method have the disadvantages of low extraction efficiency and great difference in the purity of separated sericin [20-23]. Therefore, most of the silk weaving plants treat silk reeling wastewater as waste material. In recent years, it is believed that sericin has important biological characteristics, such as corrosion resistance, antibacterial activity, and ultraviolet radiation (UV) protection [24-27]. Sericin contains a large amount of serine and a high proportion of several polar amino acids, making it highly hydrophobic and adhesive and easy to absorb and release moisture content. Therefore, sericin is widely used in the fields of biomedicine, cosmetics, and the food industry. Many researchers at home and abroad have been looking for other ways to obtain sericin, and sericin cocoon is a new kind of material developed along with the development of biogenetic engineering. Through the mutant genes in silkworm cocoons, posterior silk glands of silkworms do not develop (or they degenerate), and the middle silk glands expand, so that the silkworms are bred into the sericin cocoon species that only secrete sericin. Sericin fibers spun by silkworms form sericin cocoons, with the content of sericin up to $98.5 \%$ [28], which can be directly used to extract sericin of high purity and quality. 
As a kind of protein material secreted by silkworm, a sericin cocoon has some similarities and differences with common Bombyx mori cocoons in morphology, structure, chemical properties, and physical and mechanical properties, and so on. In this paper, sericin cocoons are studied, the morphological structure characteristics and properties of sericin cocoons and common Bombyx mori cocoons (B. cocoons) are compared and analyzed through such modern test techniques as SEM, EDX, FTIR, and XRD, and the sericin is subjected to qualitative research for the moisture absorption and anti-UV properties to provide a fundamental basis for the effective utilization of sericin cocoon products in such fields as textile modification, the food industry, and cosmetics.

\section{Materials and Methods}

\subsection{Materials}

Sericin cocoons and B. cocoons were supplied by the Sericultural Research Institute, Chinese Academy of Agricultural Sciences (Zhenjiang, China), other chemicals used for the study were purchased from Shanghai Chemistry Reagent Co., Ltd. (Shanghai, China), and polyester taffeta fabrics (210T) were provided by South-East Sea Textile Co., Ltd. (Jiangsu, China).

\subsection{Morphological Structure Test}

Digital imaging equipment was used to obtain the appearance images of sericin cocoons and B. cocoons. SU8010 scanning electron microscope (Hitachi, Tokyo, Japan) was used for the morphological structure analysis of gold-sprayed cocoons under the voltage of $5 \mathrm{kV}$. The Smart EDX energy spectrometer was used to characterize and perform quantitative analysis on the samples to determine the elemental composition of the particles on the surface of the materials. The working distance of energy spectrum analysis was $8.5 \mathrm{~mm}$.

\subsection{Porosity of Cocoon}

The density $(\rho c)$ of each cocoon shell specimen can be calculated based on the weight, area, and thickness of the cocoon strip specimen. The density ( $\rho f$ ) test of silk fiber and sericin fiber is based on the Liquid Pycnometer Method in GB/T 1,033.1-2,008 (China). The porosity of the cocoon shell specimen was calculated by the formula $(P)=1-\rho c / \rho f$.

\subsection{Amino Acid Analysis}

The sericin cocoon and common cocoon specimens (dried and accurately weighed) were sealed in tubes in $6 \mathrm{~mol} / \mathrm{L} \mathrm{Hcl}$ in vacuum, hydrolyzed for $24 \mathrm{~h}$ at $(110 \pm 2)^{\circ} \mathrm{C}$, filtered, adjusted to the preset volume, and tested for amino acid composition and content with a Hitachi 835-50 automatic amino acid analyzer.

\subsection{Thermogravimetric Analysis}

Thermogravimetric analysis of cocoon materials was determined on a STA449 F3 thermogravimetric analyzer
(NETZSCH, Selb, Germany), at a heating rate of $10^{\circ} \mathrm{C} / \mathrm{min}$ and a nitrogen flow rate of $20 \mathrm{~mL} / \mathrm{min}$.

\subsection{Infrared Spectra Analysis}

The conformation of the two cocoon materials was tested by a U.S. Nicolet iS5 Fourier transform infrared spectrometer (FT-IR) under the test conditions of room temperature, the potassium bromide pressed-disk technique, and the wave number range of 400 to $4,000 \mathrm{~cm}^{-1}$. Absorption spectra were obtained by transformation using OMNIC 6.0 (Nicolet Instrument Co., USA) software.

\subsection{X-Ray Diffraction Analysis}

The X-ray spectra of the two cocoon materials were determined by a Bruker D8 advance X-ray diffractometer under the test conditions of: Ni filtering, $\mathrm{Cu}$ target Ka ray, tube voltage of 4.0 $\mathrm{kV}$, tube current of $35 \mathrm{~mA}$, scanning speed of $5^{\circ} / \mathrm{min}$, and $2 \theta$ in the range of $10^{\circ}$ to $80^{\circ}$ ).

Jade software was used for whole pattern fitting of XRD diffraction peaks in the test range, and pseudo-Voigt function was used to describe the diffraction peaks; and a straight-line background was used for peak searching and fitting. Finally, the integral area values of crystalline peaks and noncrystalline peaks were obtained. The relative crystallinity was calculated with the following formula:

$\varepsilon=\frac{\mathrm{Ic}}{\mathrm{Ic}+\mathrm{Ia}} \times 100 \%$

where Ic is the integrated intensity of crystalline peaks and la is the integrated intensity of noncrystalline peaks. During calculation, the diffraction peaks with a full width at half the maximum (FWHM) greater than $3^{\circ}$ were identified as noncrystalline peaks.

\subsection{Sericin Extraction}

Sericin cocoon and B. cocoon materials were treated in a $\mathrm{Na}_{2} \mathrm{CO}_{3}$ solution with a concentration of $0.5 \%$ at $80^{\circ} \mathrm{C}$ to $90^{\circ} \mathrm{C}$ for 40 to $50 \mathrm{~min}$. The ratio of $\mathrm{Na}_{2} \mathrm{CO}_{3}$ solution to cocoon material was $30: 1$ [20, 21]. Sericin liquids were recovered separately and were then dried to extract solid sericin particles.

\subsection{Coating Finish}

The two kinds of solid sericin particles were dissolved in deionized water at a mass ratio of 1:20 to form a sericin mixture. Hand sample machine for coating finish (Nantong Baolai Textile Equipment Co., Ltd., Nantong, China) was used for coating finish of polyester taffeta fabrics. The coating area was $300 \times 400 \mathrm{~mm}$ and the coating thickness was $1 \mathrm{~mm}$. After the coating was finished, the fabrics were placed in a constant temperature oven $\left(\right.$ at $90^{\circ} \mathrm{C}$ ) for drying for 1 hour. 


\subsection{Anti-UV Performance Test}

The anti-UV performance index (UPF) of each coated polyester base fabric was determined on a YG902C anti-UV tester according to GB/T18830 (China), and the transmittance of the base fabric was determined according to the ultraviolet radiation in the sunlight. The UVA transmittance T(UVA) was tested in the wavelength range of $315 \mathrm{~nm}$ to $400 \mathrm{~nm}$, the UVB transmittance $T(U V B)$ was tested in the wavelength range of $290 \mathrm{~nm}$ to $315 \mathrm{~nm}$, and UPF was calculated according to the formula

$$
T(U V A)_{i}=\frac{1}{m} \sum_{\lambda=315}^{400} T_{i}(\lambda)
$$

$$
\begin{aligned}
& T(U V B)_{i}=\frac{1}{k} \sum_{\lambda=290}^{315} T_{i}(\lambda) \\
& U P F_{i}=\frac{\sum_{\lambda=290}^{\lambda=400} E(\lambda) \times \varepsilon(\lambda) \times \Delta \lambda}{\sum_{\lambda=290}^{\lambda=400} E(\lambda) \times T_{i}(\lambda) \varepsilon(\lambda) \times \Delta \lambda}
\end{aligned}
$$

where $T_{i}(\lambda)$ is the spectral transmittance of sample $i$ at the wavelength of $\lambda$, and $m$ and $k$ are the measurements obtained in $315 \mathrm{~nm}$ to $400 \mathrm{~nm}$ and $290 \mathrm{~nm}$ to $315 \mathrm{~nm}$ respectively. $\lambda$ is the solar spectral irradiance in $\mathrm{w} \cdot \mathrm{m}^{-2} \cdot \mathrm{nm}^{-1}, \varepsilon(\lambda)$ is a relative erythema effect, and $\Delta \lambda$ is the wavelength interval in $\mathrm{nm}$.

\section{Results}

3.1. Comparison Between Appearances and Components of Sericin Cocoons and B. Cocoons

As can be seen from Figure 1, silkworms spin sericin only due to the characteristics of silk glands of sericin silkworms, the formed cocoon layer is thin, and because there is no fibroin in the middle of the silk as a support, the cocoon shell is brittle, and it is easy to tear the sericin cocoon by gently pulling with your hands. Therefore, there are few complete sericin cocoons
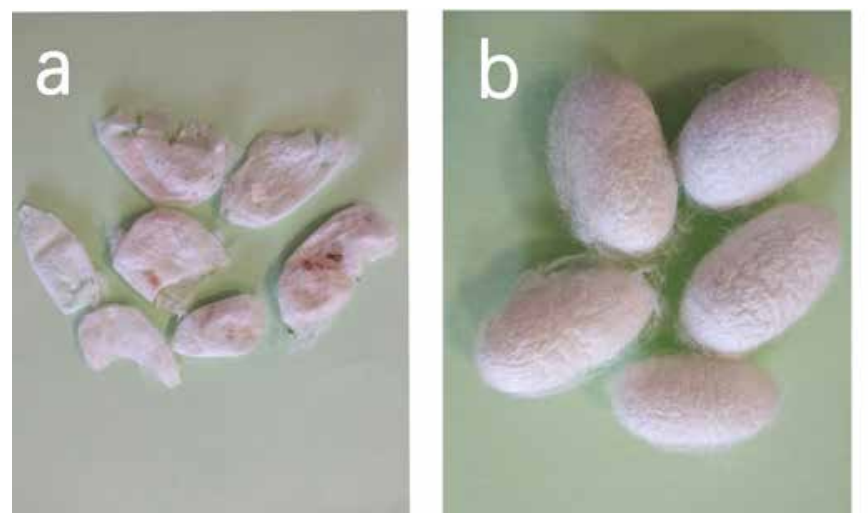

Figure 1. Appearances of sericin cocoons and common cocoons. (a) Sericin cocoon; (b) B. cocoon due to the small amount of sericin and the creeping motion of silkworm in the cocoon shell.

Figure $2 \mathrm{~A}$ is an SEM image of the outer surface of sericin cocoon. It can be seen that its morphology is similar to the appearance of $\mathrm{B}$. cocoons (Figure $2 \mathrm{C}-\mathrm{e}$ ), also belonging to the cocoon coat of the cocoon. The silk on the outer surface is irregularly entangled with itself in various forms such as twisting, turning, and interlacing, and the fibers are fluffy and disordered, the sericin of the sericin silkworm also presents distinctively fibrous, two sericin fibers extruded from the silkworm spinning mouth are combined into one, the surfaces of the sericin fibers are smooth with obvious stripes, the base fibers are densely bonded, and the pores are less than those of B. cocoons. The density of silk fibers $(\rho f)$ in sericin cocoons and B. cocoons measured by Liquid Pycnometer Method are 1,088 $\mathrm{kg} / \mathrm{m}^{3}$ and $1,317 \mathrm{~kg} / \mathrm{m}^{3}$ respectively. According to the formula for the calculation of porosity, their porosity is $58.2 \%$ and $71.3 \%$ respectively, which is basically consistent with the appearance and morphology shown in SEM images. The fiber cross-section size of the outer and inner layers of sericin cocoon is smaller than that of B. cocoons, the fine unevenness is higher, and there are some granular substances on the fibers. As can be seen from the EDX analysis results (Figure 3 ), sericin cocoons and $B$. cocoons contain trace amounts of calcium, so it can be inferred that these granular substances are calcium oxalate or other calcium salts [9]. Multiple fiber ends can be seen at the arrow in Figure 2A-a, mainly due to the fact that sericin fibers are brittle, hard, and prone to fiber fracture, while $B$. cocoon fibers are unaffected by such a problem. Figure $2 B$ is an SEM image of the inner surface of a sericin cocoon. It can be seen that the structure of the sericin cocoon is relatively compact, smooth, and flat. The silk is interwoven with "Y" type, "+" type, and various cross angles. The fibers are bonded to each other so that the cocoon layer has almost no pores, which is in line with the characteristics of silkworm cocoon lining, while the inner surface of $B$. cocoon has more pores, and also due to the sericin fibers is brittle and hard: multiple cracks in the sericin cocoon layer can be seen at the arrow in Figure 2B-c, which is also the reason why it is difficult for the sericin cocoon to maintain a complete shape, while B. cocoons are unaffected by such a problem. The inner surfaces of sericin cocoons and B. cocoons contain fewer mineral particles.

It can be seen from the amino acid analysis results in Figure 4 that sericin cocoons and B. cocoons contain the same types of amino acids, but the contents of the various amino acids are quite different. Among them, the contents of Gly, Ala, and Met in $\mathrm{B}$. cocoons are significantly higher than those in sericin cocoons, which are $2.75,5.08$, and 9.80 times higher than sericin cocoon, respectively. The contents of Asp, Thr, Glu, Lys, His, and Arg in sericin cocoons were significantly higher than those in B. cocoons, which were 3.57, 3.42, 3.29, 3.30, 2.32 , and 5.17 times higher than $B$. cocoons, respectively. The content of the different types of amino acids determines the differences in the properties of sericin and silk fibroin, especially the difference in solubility. There are a large number of polar hydrophilic side groups in amino acids, such as Asp, Thr, Ser, and Glu, which are rich in sericin. These polar hydrophilic side groups are exposed to the surface of molecules to form 


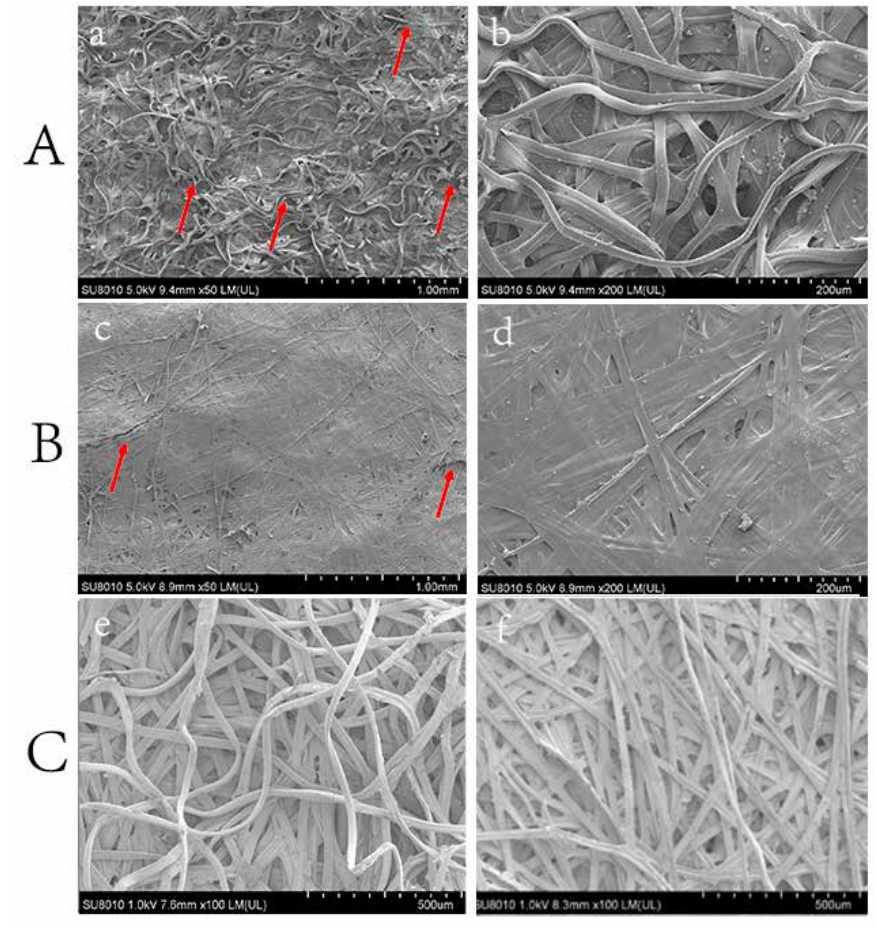

Figure 2. SEM images of sericin cocoons and B. cocoons. A. SEM images of outer surface of a sericin cocoon (a-50x, b-200x) B. SEM images of inner surface of sericin cocoon (c-50x, d-200x) C. SEM images of the outer and inner layers of a B. cocoon (e-outer surface, 100 $x$, f-inner surface, $100 \mathrm{x}$ )
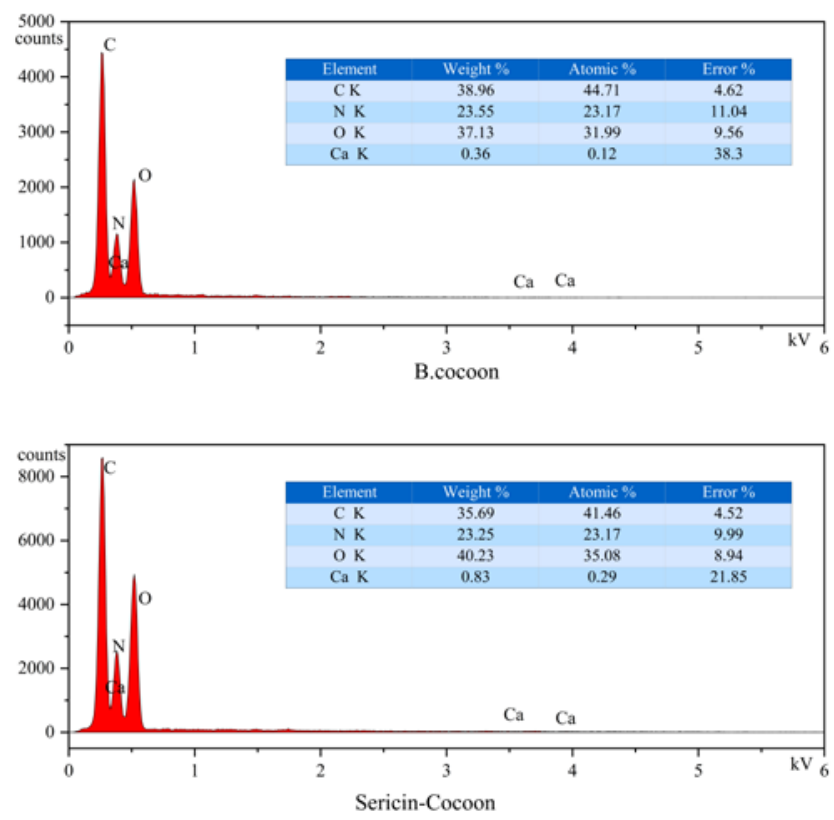

Figure 3. EDX energy spectrum diagram of a sericin cocoon and a B. cocoon

hydrophilic shells, showing good hydrophilicity and ease of dissolution in water.

\subsection{Comparative Analysis of the Thermal Properties of Sericin Cocoons and B. Cocoons}

Figure 5 shows the thermal decomposition process of sericin cocoons and $B$. cocoons. Both materials have similar thermal behavior. TG curves show that obvious dehydration and weight

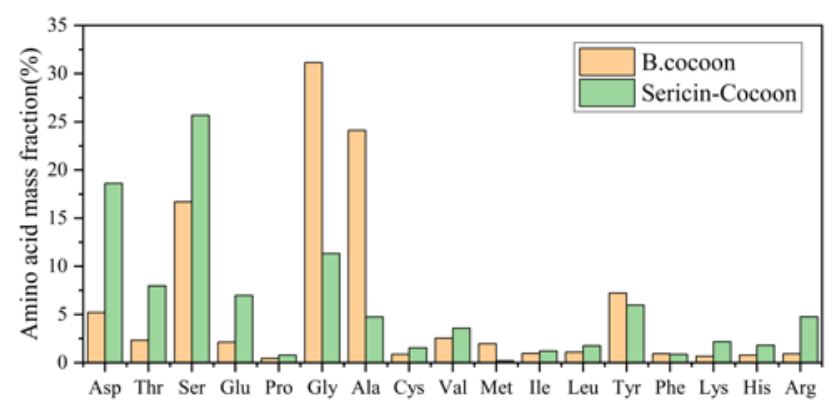

Figure 4. Amino acid analysis diagram of sericin cocoons and B. cocoons

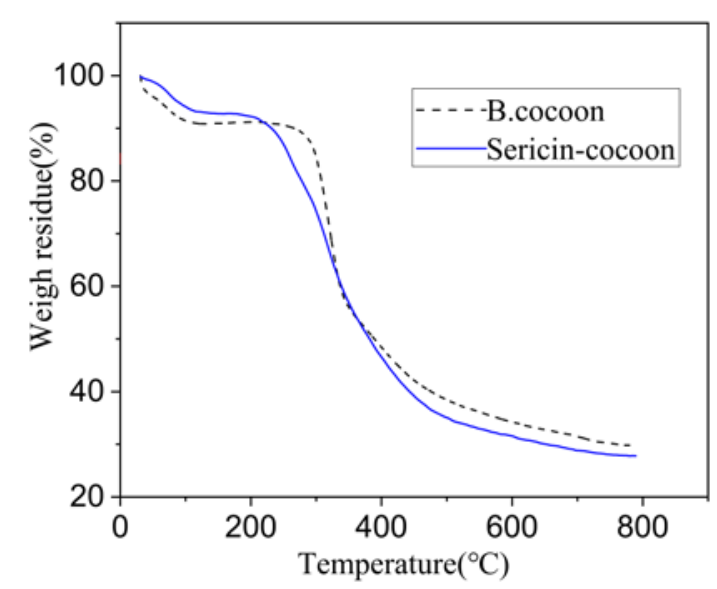

Figure 5. TG curves of sericin cocoons and B. cocoons

loss temperatures appear at $129^{\circ} \mathrm{C}$ and $111^{\circ} \mathrm{C}$, respectively, due to the moisture content of sericin cocoons and $\mathrm{B}$. cocoons, and the water molecule weight loss rates are $6.9 \%$ and $9.1 \%$, respectively, indicating that a sericin cocoon has good water retention capacity, but the water physically absorbed is less because of its lower porosity. Then there is a period of mass stability, and as the temperature continues to rise, weight loss is relatively slow. The severe weight loss of sericin cocoons and B. cocoons occurs at the temperatures of $203^{\circ} \mathrm{C}$ to $474^{\circ} \mathrm{C}$ and $267^{\circ} \mathrm{C}$ to $454^{\circ} \mathrm{C}$, respectively. The temperature range of severe decomposition of common cocoons is small, and then as the temperature continues to rise, there is also obvious weight loss until the set temperature is reached. The weight loss rates of sericin cocoons and B. cocoons are $72.2 \%$ and $70.3 \%$, respectively. The weight loss rate of $\mathrm{B}$. cocoons is lower than that of sericin cocoons, which should be related to the presence of fibroin in B. cocoons.

\subsection{Comparative Analysis of Infrared Spectra of Sericin Cocoons and B. Cocoons}

Figure 6 shows infrared spectra of sericin cocoons and B. cocoons. It can be seen that the peak shapes and peak positions of the infrared spectra of the two materials are generally similar, but there are some local differences, which have some typical characteristic peaks. Sericin cocoons and $B$. cocoons have strong absorption peaks in the vicinity of $3,267.78$ and $3,289.33 \mathrm{~cm}^{-1}$, respectively, which is mainly due to the stretching vibration of $\mathrm{O}-\mathrm{H}$ and $\mathrm{N}-\mathrm{H}$ in protein 


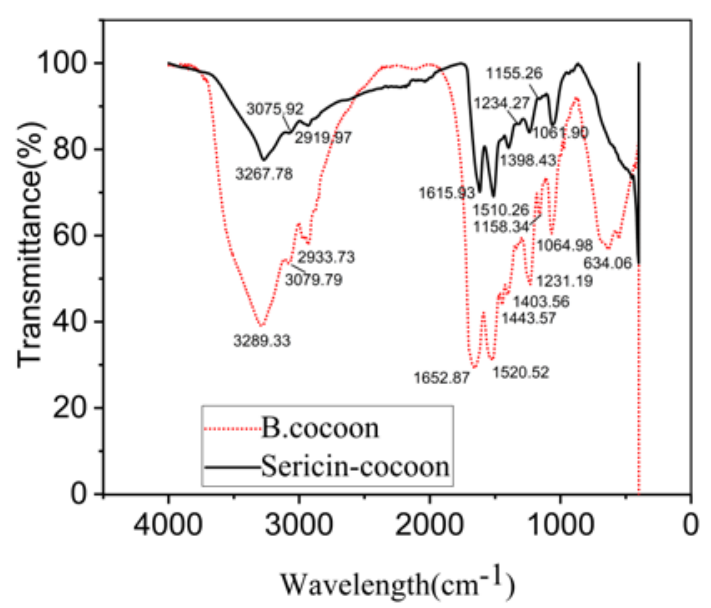

Figure 6. Infrared spectra of sericin cocoons and B. cocoons

molecules, and have peaking at 2,919.97 and 2,933.73 $\mathrm{cm}^{-1}$, respectively, which is mainly due to the stretching vibration of the $\mathrm{C}-\mathrm{H}$ bond, indicating that they both contain methylene or methyl. The peaks in the vicinity of $1,615.93$ and $1,652.87$ $\mathrm{cm}^{-1}$ are protein amide I band absorption peaks; peaks at $1,510.26$ and $1,520.52 \mathrm{~cm}^{-1}$ are amide II- $\alpha$-helix structures, respectively, whereas peaks at $1,398.43$ and $1,403.56 \mathrm{~cm}^{-1}$ are the curved vibrations of the $\mathrm{C}-\mathrm{H}$ bond, peaks at $1,234.27$ and $1,231.19 \mathrm{~cm}^{-1}$ are amide III- $\beta$-sheet structures, peaks at $1,155.26$ and $1,158.34 \mathrm{~cm}^{-1}$ are amide III- $\beta$-sheet structures, and there are characteristic absorption peaks in the vicinity of $1,060 \mathrm{~cm}^{-1}$ (amide $\mathrm{V}$ - $\beta$-sheet structure). The difference is that B. cocoons have a slightly weaker absorption peak at 634.06 $\mathrm{cm}^{-1}$ (random coil), and sericin cocoons have a protein amide II band absorption peak in the vicinity of $1,510 \mathrm{~cm}^{-1}$.

\subsection{X-Ray Diffraction Comparison and Crystallinity Analysis of Sericin Cocoons and B. Cocoons}

Figure 7 shows the XRD spectra, as well as peak differentiating and imitating of sericin cocoons and $\mathrm{B}$. cocoons. The diffraction peaks in the vicinity of $11.8^{\circ}$ and $22.0^{\circ}$ are $\alpha$-helix structures, and the diffraction peaks in the vicinity of $16.5^{\circ}, 20.2^{\circ}, 24.9^{\circ}$, $30.9^{\circ}$ and $34.59^{\circ}$ are $\beta$-sheet structures $[29,30]$. It can be seen from Figure $5 \mathrm{a}$ that the XRD patterns of sericin cocoons and B. cocoons are similar in shape, indicating that there is no material difference in the main composition of the two, except that there is a difference in the content. The strongest diffraction peaks of sericin cocoon and B. cocoon appear at $19.65^{\circ}$ and $20.78^{\circ}$ respectively. The sericin cocoon has an obvious peak at $11.33^{\circ}$ and three weak peaks at $23.89^{\circ}, 40.71^{\circ}$, and $43.13^{\circ}$, and the B. cocoon has four weak peaks at $10.63^{\circ}, 29.43^{\circ}$, $38.60^{\circ}$, and $52.84^{\circ}$, indicating that both cocoon materials have $\alpha$-helix and $\beta$-sheet structures, and the $\beta$-sheet structure is dominant. According to formula (1), the crystallinity of sericin cocoons is $10.48 \%$, and that of $B$. cocoons is $27.10 \%$. The crystallinity of $\mathrm{B}$. cocoons is higher than that of sericin cocoons, which is in close relation to the composition and content of the two different cocoon materials. The B. cocoon contains $80 \%$ to $85 \%$ of fibroin, with a large number of shaped areas with regular structure [30].

\subsection{Comparison of Anti-UV Properties of Sericin Cocoons and B. Cocoons}

Figure 8 shows the anti-UV performance curves of sericin cocoons and B. cocoons. Our calculation of the anti-UV performance index (UPF) of uncoated base fabric is $8.9 \%$, and UVA and UVB transmittance T(UVA) and T(UVB) are 16.8\% and $7.1 \%$, respectively; the anti-UV performance index (UPF) of base fabric coated with sericin of $B$. cocoons is $22.5 \%$, and

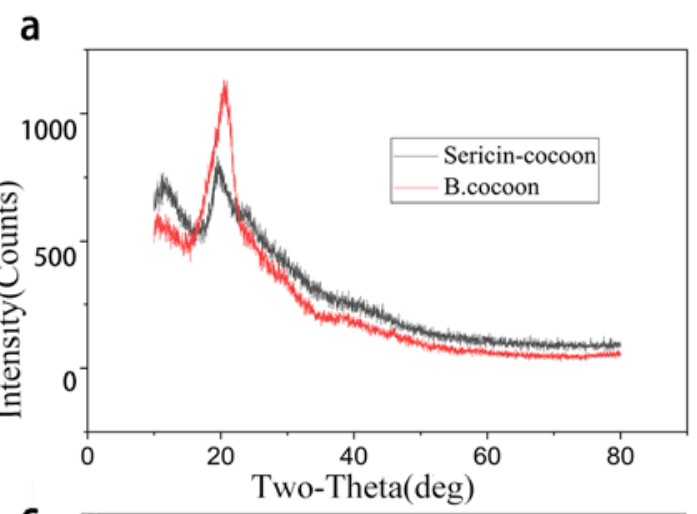

b
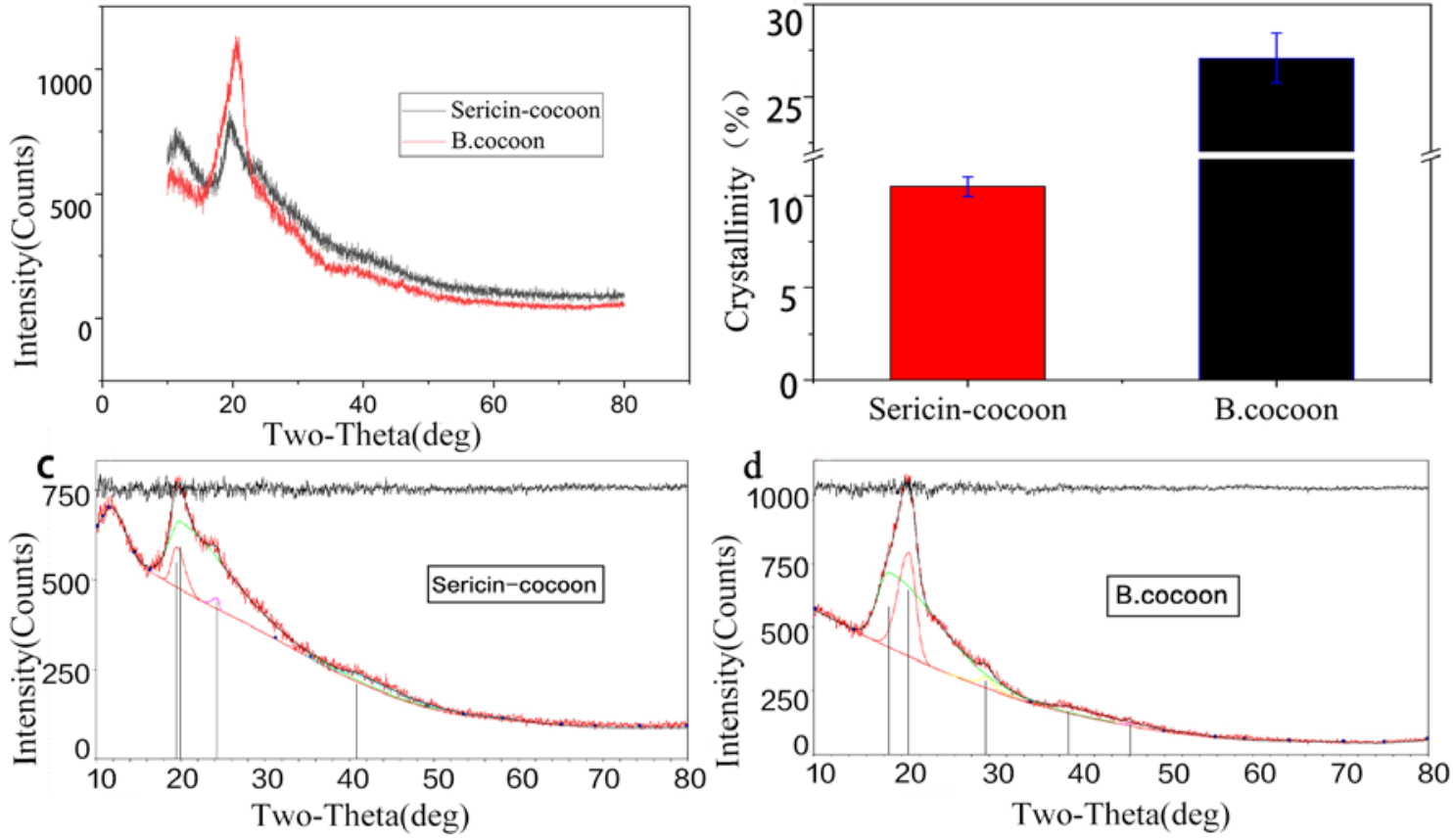

Figure 7. XRD spectra and peak-differentiating and imitating of sericin cocoons and B. cocoons. (a) XRD spectra of sericin cocoons and B. cocoons, (b) Peak-differentiating and imitating of sericin cocoons, (c)Peak differentiating and imitating of B. cocoons 


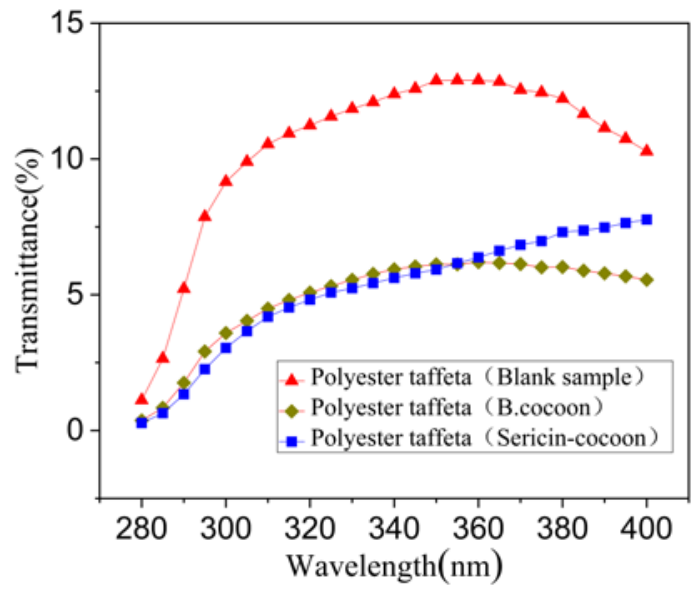

Figure 8. Anti-UV properties of sericin cocoons and B. cocoons

UVA and UVB transmittance T(UVA) and T(UVB) are $8.36 \%$ and $2.39 \%$, respectively; and the anti-UV performance index (UPF) of base fabric coated with sericin of sericin cocoon is 23.9\%, and UVA and UVB transmittance T(UVA) and T(UVB) are $6.1 \%$ and $2.22 \%$, respectively. The base fabric coated with sericin shows good anti-UV performance, which fully indicates that sericin can provide good anti-UV performance. The laws of shielding ultraviolet rays of the two base fabrics with different coatings are similar, and the anti-UV effect of the fabric coated with sericin of sericin cocoons is slightly better than that of the fabric coated with sericin of $B$. cocoons.

\section{Conclusions}

Sericin, as a biological source, can be used in such fields as product modification, food, and medicine due to its excellent biological characteristics, with unique antibacterial activity, ultraviolet protection, high hydrophilicity and adhesion, and the fact that it is easy to absorb and release moisture content. The development of sericin cocoons through biogenetic engineering is conducive to the extraction of sericin with high purity and high quality. In this paper, the morphology, structure, thermal properties, and anti-UV properties of sericin cocoons are compared and studied. It has been found that sericin cocoons are formed by silkworms in a normal process of silk spinning and cocooning. Although it has a light and thin cocoon layer, the sericin cocoon has the basic morphology and structure of cocoon layer of common cocoon. Because of the brittleness and hardness of sericin fibers, the sericin cocoon has poor integrity. TG analysis shows that the thermal decomposition rate of sericin cocoons is higher than that of $B$. cocoons, due to the absence of fibroin in the sericin cocoons. The experimental results of FTIR and XRD show that the two materials contain similar groups or functional groups, with both $\alpha$-helix and $\beta$-sheet structures, and dominantly the $\beta$-sheet structure. The crystallinity of sericin cocoons $(10.48 \%)$ is lower than that of B. cocoons (27.10\%). Sericin has excellent anti-UV properties. In the experiment, the anti-UV performance index (UPF) of the base fabric coated with sericin is 2 to 2.5 times that of the original base fabric. The in-depth study of sericin cocoons is conducive to expanding its application to make full use of its excellent biological properties and can provide a fundamental basis for the research of biological engineering materials based on fibroin.

Author Contributions: B.Z. conceived and designed the experiments; B.Z. and H.W. performed the experiments; H.Z. and J.Z. analyzed the data; J.Z. and J.C. contributed reagents/materials/analysis tools; $B . Z$. wrote and submitted the manuscript. All authors have read and agreed to the published version of the manuscript.

Funding: This research was funded by the Construction Project of Integrated Platform for Integration of Industry and Education in Higher Vocational Education in Jiangsu Province, grant number Education Department of Jiangsu Province (2019) 26, Jiangsu Province Higher Vocational Education High-level Major Group Construction Project-Modern Textile Technology Major Group, 2020 Jiangsu Province Higher Vocational Colleges Teachers Professional Leaders high-end Research (team visit research), 2020 Jiangsu productionstudy-research Cooperation Project (BY2020336) Qinglan Project of Educational Department of Jiangsu Province, grant number Education Department of Jiangsu Province (2019)3. General Program of Natural Science Foundation of Jiangsu Province (BK20201216).

Conflicts of Interest: The authors declare no conflict of interest.

\section{References}

[1] Chen, F., Porter, D., Vollrath, F. (2012). Morphology and structure of silkworm cocoons. Materials Science \& Engineering C-Materials for Biological Applications, 32(4), 772-778.

[2] Zhang, J., Kaur, J., Rajkhowa, R., Li, J. L., Liu, X. Y., Wang, $X$. G. (2013). Mechanical properties and structure of silkworm cocoons: A comparative study of Bombyx mori, Antheraea assamensis, Antheraea pernyi and Antheraea mylitta silkworm cocoons. Materials Science \& Engineering C-Materials for Biological Applications, 33(6), 3206-3213.

[3] Chen, F., Hesselberg, T., Porter, D., Vollrath, F. (2013). The impact behaviour of silk cocoons. Journal of Experimental Biology, 216(14), 2648-2657.

[4] Chen, F., Porter, D., Vollrath F. (2012) Structure and physical properties of silkworm cocoons. Journal of the Royal Society Interface, 9(74), 2299-2308.

[5] Chen, F., Porter, D., Vollrath, F. (2010). Silkworm cocoons inspire models for random fiber and particulate composites. Physical Review E, 82(4).

[6] Jin, X., Zhang, J., Gao, W., Li, J., Wang, X. (2014). Cocoon of the silkworm Antheraea pernyi as an example of a thermally insulating biological interface. Biointerphases, 9(3).

[7] Zhang, J., Rajkhowa, R., Li, J. L., Liu, X. Y., Wang, X. G. (2013). Silkworm cocoon as natural material and structure for thermal insulation. Materials \& Design, 49, 842-849.

[8] Xiao, X., Chen, C., Liu, W., Zhang, Y. (2017). Structure, features and biomedical applications of silk sericin. Progress in Chemistry, 29(5), 513-523. 
[9] Pastero, L., Aquilano, D. (2018). Calcium carbonate polymorphs growing in the presence of sericin: A new composite mimicking the hierarchic structure of nacre. Crystals, 8(7).

[10] Wang, Y.-J., Zhang, Y.-Q. (2011). Three-layered sericins around the silk fibroin fiber from Bombyx mori cocoon and their amino acid composition. In: Bai, L., Chen, G. Q. (Eds.) Silk: Inheritance and Innovation - Modern Silk Road. Vol 175-176,

[11] Gheysens, T., Collins, A., Raina, S., Vollrath, F., Knight, D. $P$. (2011). Demineralization enables reeling of wild silkmoth cocoons. Biomacromolecules, 12(6), 2257-2266.

[12] Wang, T., et al. (2017). Structure and properties of silkworm cocoon (Bombyx mori) treated by hot pressing. Materials \& Design, 134:132-138.

[13] Chen, F., Porter, D., Vollrath, F. (2012). Silk cocoon (Bombyx mori): Multi-layer structure and mechanical properties. Acta Biomaterialia, 8(7), 2620-2627.

[14] Dutta, S., Talukdar, B., Bharali, R., Rajkhowa, R., Devi, D. (2013). Fabrication and characterization of biomaterial film from gland silk of muga and eri silkworms. Biopolymers, 99(5), 326-333.

[15] Mazzi, S., Zulker, E., Buchicchio, J., Anderson, B., Hu, X. (2014). Comparative thermal analysis of Eri, Mori, Muga, and Tussar silk cocoons and fibroin fibers. Journal of Thermal Analysis and Calorimetry, 116(3), 1337-1343.

[16] Reddy, N., Zhao, Y., Yang, Y. (2013). Structure and properties of cocoons and silk fibers produced by Attacus atlas. Journal of Polymers and the Environment, 21(1),1623.

[17] Reddy, N., Yang, Y. (2010). Structure and properties of cocoons and silk fibers produced by Hyalophora cecropia. Journal of Materials Science, 45(16), 4414-4421.

[18] Zhou, B., Wang, H., Zhou, H., Wang, K., Wang, S. (2020). Natural flat cocoon materials constructed by eri silkworm with high strength and excellent anti-ultraviolet performance. Journal of Engineered Fibers and Fabrics, 15.

[19] Du, X., Li, J., Chen, Y. (2011) Proteomic analysis of sericin in Bombyx mori cocoons. Biotechnology and Bioprocess Engineering, 16(3), 438-444.

[20] Zhao, C.-Y., Wang, Y., Li, Y.-F., Chen, Z.-H. (2015). Optimization of extraction technology for sericin from silkworm cocoon with orthogonal design. Zhong yao cai = Zhongyaocai $=$ Journal of Chinese Medicinal Materials, 38(5), 915-918.
[21] Cao, T.-T., Wang, Y.-J., Zhang, Y.-Q. (2014). Silk reeling of silkworm cocoon in strongly alkaline electrolyzed water as a sericin swelling agent at low temperature. Journal of the Textile Institute, 105(5), 502-508.

[22] Capar, G. (2012). Separation of silkworm proteins in cocoon cooking wastewaters via nanofiltration: Effect of solution $\mathrm{pH}$ on enrichment of sericin. Journal of Membrane Science, 389, 509-521.

[23] Rocha $L K H$, et al. (2017). Sericin from Bombyx mori cocoons. Part I: Extraction and physicochemical-biological characterization for biopharmaceutical applications. Process Biochemistry, 61, 163-177.

[24] Wang, Y., Zhang, C.-H., He, J.-H., Xu, W.-L., Liu, X. (2019). UV Protection of waterborne polyurethane films by silk sericin incorporation. In: Li, Y., Zhang, K., Pan, Z., Li, G. (Eds.) Textile Bioengineering and Informatics Symposium. 118-123.

[25] Saravanan, A., Huang, B.-R., Kathiravan, D. (2020). Enhancement of UV photodetection properties of hierarchical core-shell heterostructures of a natural sericin biopolymer with the addition of $\mathrm{ZnO}$ fabricated on ultrananocrystalline diamond layers. Acs Applied Materials \& Interfaces, 12(2), 3,254-3,264.

[26]. Zhang, J., Li, J., Jin, X., Du, S., Kaur, J., Wang, X. (2017). Natural and highly protective composite structures - Wild silkworm cocoons. Composites Communications, 4:1-4.

[27] Roy, M., et al. (2012). Carbondioxide gating in silk cocoon. Biointerphases, 7(1-4).

[28] Zhang, X., Khan, M. M. R., Yamamoto, T., Tsukada, M., Morikawa, H. (2012). Fabrication of silk sericin nanofibers from a silk sericin-hope cocoon with electrospinning method. International Journal of Biological Macromolecules, 50(2), 337-347.

[29] Sampath, S., et al. (2012). X-ray diffraction study of nanocrystalline and amorphous structure within major and minor ampullate dragline spider silks. Soft Matter, 8(25), 6713-6722.

[30] Balcao, V. M., Harada, L. K., Jorge, L.R., Oliveira, Jr., J. M., Tubino, M., Vila, M. M. D. C. (2020). Structural and functional stabilization of sericin from Bombyx mori cocoons in a biopolysaccharide film: Bioorigami for skin regeneration. Journal of the Brazilian Chemical Society, 31(4), 833-848. 